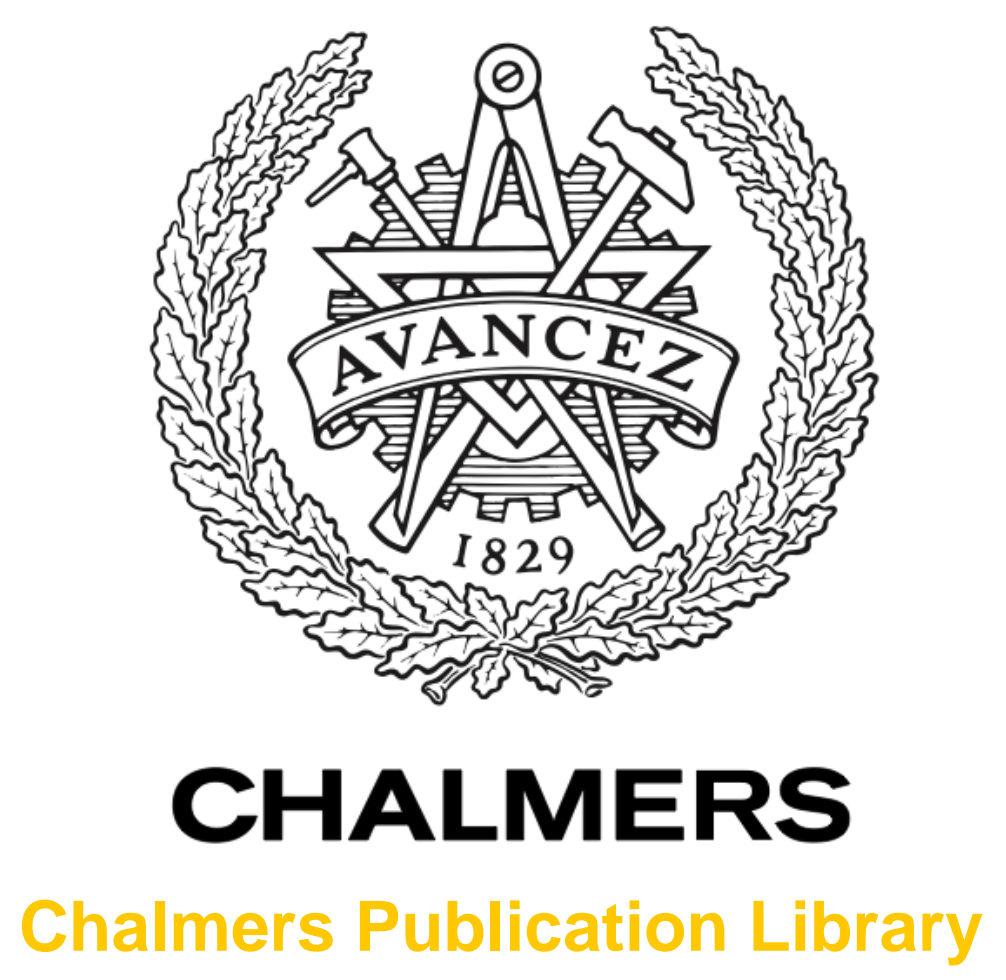

\title{
An Economic NMPC Formulation for Wind Turbine Control
}

This document has been downloaded from Chalmers Publication Library (CPL). It is the author's version of a work that was accepted for publication in:

\section{Conference on Decision and Control 2013}

Citation for the published paper:

Gros, S. (2013) "An Economic NMPC Formulation for Wind Turbine Control". Conference on Decision and Control 2013

Downloaded from: http://publications.lib.chalmers.se/publication/194411

Notice: Changes introduced as a result of publishing processes such as copy-editing and formatting may not be reflected in this document. For a definitive version of this work, please refer to the published source. Please note that access to the published version might require a subscription. 


\title{
An Economic NMPC Formulation for Wind Turbine Control
}

\author{
Sébastien Gros
}

\begin{abstract}
Model Predictive Control (MPC) is a strong candidate for the control of large Multi-MegaWatt Wind Turbine Generators. Several MPC and some Nonlinear MPC scheme have been proposed in the literature, based on referencetracking objective functions. While the resulting schemes offer very promising results, the difficulty of tuning a referencetracking NMPC scheme for performance is likely to be a hindrance to the industrial success of NMPC-based WTG control. Because they directly maximize the system performance, economic NMPC schemes require no tuning. Economic NMPC schemes present, however, some difficulties that are a serious obstacle to real-time implementations. This paper presents an economic NMPC formulation for maximizing the generated power of wind turbine generators, which does not suffer from such difficulties and is therefore well suited for its real-time deployment.
\end{abstract}

Keywords : Wind Turbine Control, economic NMPC, Power Optimization, Operational Constraints

\section{INTRODUCTION}

Model Predictive Control (MPC) techniques are emerging as strong candidates for the control of future Multi-Megawatt Wind Turbine Generators (MM-WTG) because they can handle actuator limitations, intricate operational strategies, the dynamic nonlinearities, the control allocation among multiple actuators, and the predictive information gathered through LIDAR systems [10]. Promising results have been obtained in simulations, showing the potential of MPC and Nonlinear MPC (NMPC) techniques for power generation and structural load alleviation [11], [6], [13], [7].

Most MPC schemes for WTG control rely on a referencetracking objective function, which acts as a surrogate for maximizing the power generation. In the below-rated region [3], the tracking objective function penalizes the deviation of the state from its wind-dependent steady-state optimal value. In the above-rated region, the rated values are used as references. Different weights are chosen in the different control regions [11]. The reference-tracking approach for maximizing the generated power yields good performances in practice. However, its connection to the actual goal of maximizing the power generation is very indirect.

The overall performance of the NMPC scheme is heavily dependent on the tuning of the reference-tracking objective function, and requires a delicate balance of the competing control objectives: maximizing the power capture, maximizing the constancy of the generated power, minimizing the structural fatigue and maximizing the life-time of the actuators. Such tuning is typically performed based on intricate heuristic procedures, involving time-consuming simulations

S. Gros is with the Optimization in Engineering Center (OPTEC), K.U. Leuven, Kasteelpark Arenberg 10, B-3001 Leuven-Heverlee, Belgium. sgros@esat. kuleuven.be in order to select the appropriate weights. Moreover, certifying that the resulting design is the best choice in economical terms is very difficult. Having an objective function that can be directly translated into economical terms is therefore of paramount interest for making the controller design faster and more systematic.

Using an economic objective function in the context of NMPC can present some difficulties [9], the most salient practical ones being that it can yield a turnpike which often forbids the use of a short prediction horizon, and that a Gauss-Newton Hessian approximation can often not be used. This paper, nevertheless, proposes an objective function for maximizing the power generation that does not suffer from these difficulties, and which is straightforward to translate into economical terms.

This paper is organized as follows. Section III presents a formal analysis of the power optimization problem, and develops an objective function that is arguably best suited for a real-time NMPC scheme maximizing the power generation. Section IV reformulates the power generation objective function in term of power capture, and presents a systematic way of choosing the tracking weights in the classical referencetracking scheme. Section V presents a technique to formulate the power capture problem developed in Section IV in a form suitable for a Gauss-Newton Hessian approximation, which is ideal for a real-time implementation. In Section VI the implementation of the operational constraints such as the bounds on the rotor speed and generated power in the form of relaxed constraints is discussed, and a regularization of the generator torque that can be translated into a penalty on the power quality is presented. Conclusions and future developments are discussed in Section VII. Next Section briefly presents the WTG model used in this work.

A real-time implementation of the proposed NMPC scheme is presented in [4].

\section{Wind TURBINE MODEL}

For the sake of simplicity, this paper uses a very simple WTG model, ignoring the structural dynamics, whose direct influence on the generated power is negligible. The model reads:

$$
\begin{aligned}
J \dot{\omega} & =T_{\text {aero }}-r_{\mathrm{g}}^{-1} T_{\mathrm{g}}, \\
T_{\text {aero }} & =\frac{1}{2} \rho A C_{P}(\beta, \lambda) \frac{W^{3}}{\omega}, \quad \lambda=\frac{R \omega}{W},
\end{aligned}
$$

where $\omega$ is the rotor speed, $T_{\text {aero }}$ the aerodynamic torque perceived by the rotor, $W$ is the free wind speed. Factor $\lambda$ is the tip-speed ratio, which plays an important role in the aerodynamics of WTG. Parameter $J=J_{\mathrm{r}}+r_{\mathrm{g}}^{-2} J_{\mathrm{g}}$ is the total 
TABLE I

MODEL PARAMETERS

\begin{tabular}{|c||c|l|}
\hline Parameter & Description & Value \\
\hline$J$ & Total rotor inertia & $40.47 \cdot 10^{6}\left[\mathrm{~kg} / \mathrm{m}^{2}\right]$ \\
$r_{\mathrm{g}}$ & Gearbox ratio & $1 / 97[-]$ \\
$\rho$ & Air density & $1.23\left[\mathrm{~kg} / \mathrm{m}^{3}\right]$ \\
$A$ & Rotor area & $1.25 \cdot 10^{4}\left[\mathrm{~m}^{2}\right]$ \\
$R$ & Rotor radius & $63[\mathrm{~m}]$ \\
\hline
\end{tabular}

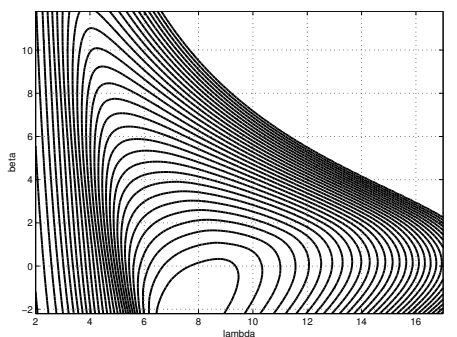

Fig. 1. Level curves of the function $C_{P}(\beta, \lambda)$ for a classical $5 \mathrm{MW}$ industrial WTG, computed using the blade-momentum model of the windturbine modeling software Bladed.

turbine inertia as seen from the rotor, where $J_{\mathrm{r}}$ is the rotor inertia, including the hub and the blades, $J_{\mathrm{g}}$ is the generator inertia, and $r_{\mathrm{g}}$ is the gearbox ratio. The state and the control input vectors $X \in \mathbb{R}^{2}$ and $U \in \mathbb{R}^{2}$ are given by:

$$
X=\left[\begin{array}{ll}
\omega & \beta
\end{array}\right], \quad U=\left[\begin{array}{ll}
\dot{\beta} & T_{\mathrm{g}}
\end{array}\right] .
$$

In the following, the dynamics (1) are lumped into the function $f$ :

$$
\dot{X}=f(X, U, W) \text {. }
$$

The aerodynamic power harvested from the wind field reads:

$$
P_{\text {aero }}=\frac{1}{2} \rho A C_{P}(\beta, \lambda) W^{3},
$$

while the generated power reads:

$$
P=\eta r_{\mathrm{g}}^{-1} T_{\mathrm{g}} \omega
$$

where $\eta<1$ is the generator efficiency. The model parameters are summarized in table II.

The maximum aerodynamic efficiency is achieved by maximizing $C_{P}$. At steady-state, it is yielded by the windindependent optimal values $\beta^{*}$ and $\lambda^{*}$ achieving the maximal value of $C_{P}$, labeled $C_{P}^{\max }$. In the following, it will be useful to substitute $\lambda$ in $C_{P}$, and therefore consider $C_{P}$ as a function of $\omega, \beta, W$. The optimal steady-state rotor speed $\omega^{*}$ is linearly dependent on the wind speed $W$, with

$$
\omega^{*}=R^{-1} \lambda^{*} W
$$

\section{POWER OPTIMIZATION}

Economic NMPC is based on repeatedly solving an optimal control problem (OCP) that best represents the control objectives. In the context of wind turbine control, a natural choice of underlying OCP seeks to maximize the energy generated over the control horizon, subject to constraints of the rotor speed $\omega$, generator torque $T_{\mathrm{g}}$, and pitch rate $\dot{\beta}$, i.e.:

$$
\begin{array}{ll}
\min _{U, X} & -\int_{t}^{t+T_{\mathrm{c}}} P d t \\
\text { s.t. } & (1), \quad-\dot{\beta}^{\max } \leq \dot{\beta} \leq \dot{\beta}^{\max }, \quad 0 \leq T_{\mathrm{g}} \leq T_{\mathrm{g}}^{\max } \\
& P \leq P^{\max }, \quad \omega^{\min } \leq \omega \leq \omega^{\max }
\end{array}
$$

This type of formulation is used in e.g. [5]. This section, however, shows the shortcomings of formulation (4), and proposes an alternative approach better suited for real-time deployments of economic NMPC.

\section{A. Analysis of the Power Optimization Problem}

This section proposes a formal analysis of problem (4). The Hamiltonian [2] of problem (4) reads:

$$
H=\mu^{T} f-P, \quad \text { with } \quad P=\eta r_{\mathrm{g}}^{-1} T_{\mathrm{g}} \omega
$$

where $\mu=\left[\begin{array}{ll}\mu_{1} & \mu_{2}\end{array}\right]$ is the vector of Lagrange multipliers. It follows that the derivative of $H$ with respect to the inputs $U$ reads:

$$
H_{U}=\left[\begin{array}{ll}
-r_{\mathrm{g}}^{-1}\left(\eta \omega+J^{-1} \mu_{1}\right) & \mu_{2}
\end{array}\right]
$$

is independent of $U$, hence problem (4) is singular. Using $\dot{\mu}^{T}=-H_{X}$, the time-derivative of $H_{U}$ then reads:

$$
\dot{H}_{U}=\frac{1}{2} \rho A \frac{W^{3}}{J \omega}\left[\begin{array}{c}
-\frac{\eta}{r_{\mathrm{g}}} C_{P}-\frac{\mu_{1}}{J r_{\mathrm{g}}}\left(\omega^{-1} C_{P}-\frac{\partial C_{P}}{\partial \omega}\right) \\
-\mu_{1} \frac{\partial C_{P}}{\partial \beta}
\end{array}\right]
$$

So that the manifold:

$$
\frac{\partial C_{P}}{\partial \beta}=\frac{\partial C_{P}}{\partial \omega}=0, \quad \eta \omega+J^{-1} \mu_{1}=0
$$

uniquely yields $H_{U}=\dot{H}_{U}=0$ (see e.g. [2], [15]). Because both inputs appear in $\ddot{H}_{U}$, the degree of singularity of problem (4) is $\sigma=2$.

In the absence of a terminal cost in (4), the terminal boundary condition for the adjoint variables is $\mu\left(t+T_{\mathrm{c}}\right)=0$ unless some constraint is activated at final time. As a result, condition (5) entails $\omega\left(t+T_{\mathrm{c}}\right)=0$ or the activation of some constraint at final time. Hence the solution to problem (4) cannot maximize $C_{P}$ toward the end of the prediction horizon. This behavior is common in economic NMPC and is labeled turnpike in the literature [9].

The behavior of the optimal solution to problem (4) has a simple physical interpretation. Because of the finite prediction horizon $T_{\mathrm{c}}$, problem (4) disregards the performance that can be achieved after $t+T_{\mathrm{c}}$. As a result, the best strategy to maximize the energy generation over the time interval $\left[t, t+T_{\mathrm{c}}\right]$ is to extract kinetic energy from the rotor toward the end of the prediction horizon, i.e. to apply a maximum generator torque $T_{\mathrm{g}}$ and reduce the rotor speed $\omega$. This physical interpretation is used in the next section to reformulate (4) and correct the turnpike. 


\section{B. Reformulation of the Power Optimization Problem}

It has been established in Section III-A that the wind turbine power optimization problem (4) must have a turnpike property, which extracts kinetic energy at the end of the prediction horizon. Based on this physical interpretation, the following correction of problem (4) is proposed:

$$
\begin{array}{ll}
\min _{U, X} & -\frac{\eta}{2} J \omega\left(t+T_{\mathrm{c}}\right)^{2}-\int_{t}^{t+T_{\mathrm{c}}} P d t \\
\text { s.t. } & (1), \quad-\dot{\beta}^{\max } \leq \dot{\beta} \leq \dot{\beta}^{\max }, \quad 0 \leq T_{\mathrm{g}} \leq T_{\mathrm{g}}^{\max } \\
& P \leq P^{\max }, \quad \omega^{\min } \leq \omega \leq \omega^{\max } .
\end{array}
$$

The adjunction of the wind turbine terminal kinetic energy $\frac{1}{2} J \omega\left(t+T_{\mathrm{c}}\right)^{2}$ in the cost function, corrected by the efficiency $\eta$ accounts for the energy stored in the rotor, and removes the incentive to extract kinetic energy at the end of the prediction horizon. This statement is formally established next.

Due to the adjunction of the terminal cost $\phi=-\frac{\eta}{2} J \omega^{2}$ to the objective function, in the absence of active constraints at final time $t+T_{\mathrm{c}}$, the boundary conditions for the adjoint variables $\mu$ read:

$$
\mu\left(t+T_{f}\right)=\frac{\partial \phi}{\partial x}\left(t+T_{\mathrm{c}}\right)=\left[\begin{array}{ll}
-J \eta \omega & 0
\end{array}\right]^{T}
$$

and therefore satisfy $H_{U}=0$. As a result, problem (6) terminates on the manifold:

$$
\frac{\partial C_{P}}{\partial \beta}=\frac{\partial C_{P}}{\partial \omega}=0, \quad \eta \omega+J^{-1} \mu_{1}=0
$$

where the maximum aerodynamic power capture is achieved.

\section{Illustrative example}

The observations reported in Sections III-A-III-B are illustrated here for a below-rated wind speed $W=8[\mathrm{~m} / \mathrm{s}]$ corresponding to an optimal generator speed of $\omega^{*}=98.63[\mathrm{rad} / \mathrm{s}]$ and an optimal generator torque of $T_{\mathrm{g}}^{*}=18.542[\mathrm{kNm}]$. Problem (4) was solved starting from $\omega(t)=0.95 \omega^{*}$. It can be seen in Fig. 2 that the minimum torque $T_{\mathrm{g}}=0$ is applied until $\omega^{*}$ is reached, at about $t=11[s]$. Then the manifold $\frac{\partial C_{P}}{\partial \beta}=\frac{\partial C_{P}}{\partial \omega}=0$ is held from $t=11[s]$ to $t=66[s]$. Finally, the maximum torque $T_{\mathrm{g}}=T_{\mathrm{g}}^{\max }$ is applied until final time is reached. The latter is labeled turnpike in the literature.

Because of the large inertia of MM-WTG, the turnpike is dramatic for short prediction horizons. It can be observed in Fig. 3 (red lines) that with a shorter prediction horizon $\left(T_{\mathrm{c}}=15[\mathrm{~s}]\right.$ ), the solution enters directly the turnpike, where the speed is reduced. Since using reasonably short prediction horizon is a key component for fast NMPC, the objective function in problem (4) is detrimental for a real-time deployment of the NMPC scheme.

The effect of introducing the terminal cost $\phi=-\frac{\eta_{\mathrm{g}}}{2} J \omega^{2}$ in the cost function is illustrated in Fig. 3, where the same wind speed and initial conditions were used as in Fig. 2, with a prediction horizon $T_{\mathrm{c}}=15[\mathrm{~s}]$. It can be seen that problem (6) terminates at the optimal generator speed $\omega\left(t+T_{\mathrm{c}}\right)=\omega^{*}=$ $98.63[\mathrm{rad} / \mathrm{s}]$.
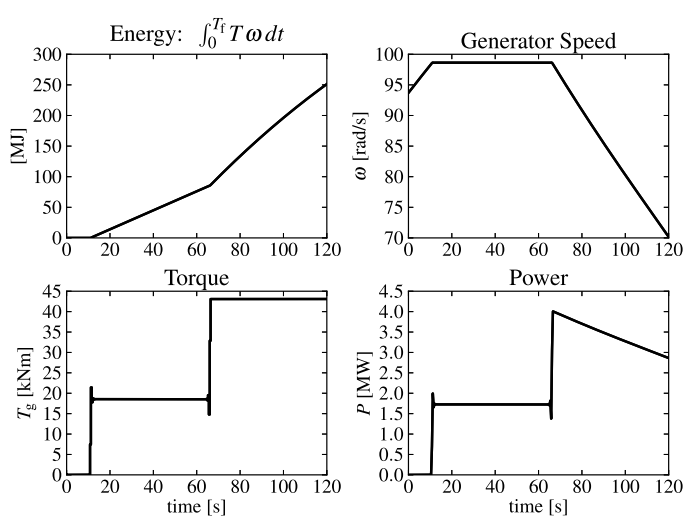

Fig. 2. Turnpike for a prediction horizon $T_{\mathrm{c}}=120[\mathrm{~s}]$. The solution for optimal power generation cannot terminates on the manifold $\frac{\partial C_{P}}{\partial \omega}=0, \frac{\partial C_{P}}{\partial \beta}=$ 0 .
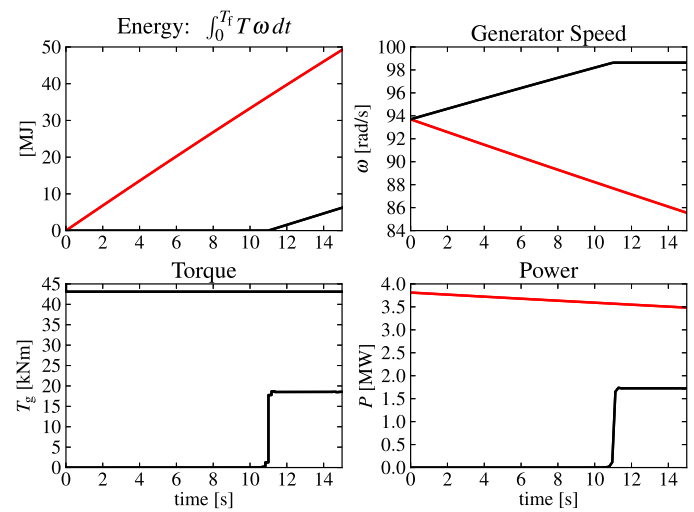

Fig. 3. Corrected Turnpike for a prediction horizon $T_{\mathrm{c}}=15[\mathrm{~s}]$. For a short prediction horizon, the solution for optimal power generation problem (4) enters directly the last phase of the solution with $T=T_{\max }$ (red lines), while the solution corrected problem (6) terminates on the manifold $\frac{\partial C_{P}}{\partial \omega}=$ $0, \frac{\partial C_{P}}{\partial \beta}=0$ (black lines).

\section{Power Optimization \& REFEREnCE Tracking}

This section reformulate problem (6) as a maximization of the power capture, and establishes its connection with reference-tracking schemes for WTG control.

\section{A. Equivalent cost function for power optimization}

Ignoring the variations resulting from the tower fore-aft oscillations, the energy captured from the wind field by the WTG in the time interval $\left[t, t+T_{\mathrm{c}}\right]$ reads:

$$
E_{\text {wind }}=\int_{t}^{t+T_{\mathrm{c}}} P_{\text {aero }} d \tau=\frac{1}{2} \rho A \int_{t}^{t+T_{\mathrm{c}}} C_{P} W^{3} d \tau
$$

and is distributed between the kinetic energy stored in the rotor and the energy extracted from the rotor by the generator, i.e. $E_{\text {wind }}=E_{\text {kin }}+E_{\text {gen }}$ with:

$$
E_{\mathrm{kin}}=\frac{1}{2} J\left(\omega\left(t+T_{\mathrm{c}}\right)^{2}-\omega(t)^{2}\right), \quad E_{\mathrm{gen}}=\int_{t}^{t+T_{\mathrm{c}}} r_{\mathrm{g}}^{-1} T_{\mathrm{g}} \omega d \tau .
$$

It follows that the cost function in (6):

$$
\Phi_{0}=-\eta\left(\frac{1}{2} J \omega\left(t+T_{\mathrm{c}}\right)^{2}+\int_{t}^{t+T_{\mathrm{c}}} r_{\mathrm{g}}^{-1} T_{\mathrm{g}} \omega d \tau\right)
$$


TABLE II

MODEL PARAMETERS

\begin{tabular}{|c||c|l|}
\hline Parameter & Description & Value \\
\hline$J$ & Total rotor inertia & $40.47 \cdot 10^{6}\left[\mathrm{~kg} / \mathrm{m}^{2}\right]$ \\
$r_{\mathrm{g}}$ & Gearbox ratio & $1 / 97[-]$ \\
$\rho$ & Air density & $1.23\left[\mathrm{~kg} / \mathrm{m}^{3}\right]$ \\
$A$ & Rotor area & $1.25 \cdot 10^{4}\left[\mathrm{~m}^{2}\right]$ \\
$R$ & Rotor radius & $63[\mathrm{~m}]$ \\
$\eta$ & Generator/gear-box efficiency & $0.95[-]$ \\
\hline
\end{tabular}

is equivalent to:

$$
\Phi_{0}=-\eta\left(E_{\mathrm{kin}}+E_{\mathrm{gen}}\right)-\frac{\eta}{2} J \omega(t)
$$

Since $\omega(t)$ is fixed by the state of the wind turbine at time $t$ and since $\eta$ is a constant, it follows that maximizing $\Phi_{0}$ is equivalent to maximizing the aerodynamic power capture, i.e. (6) can be rewritten as:

$$
\begin{array}{ll}
\min _{U, X} & -\frac{1}{2} \rho A \int_{t}^{t+T_{\mathrm{c}}} C_{P} W^{3} d \tau \\
\text { s.t. } & (1), \\
& -\dot{\beta}^{\max } \leq \dot{\beta} \leq \dot{\beta}^{\max }, \quad 0 \leq T_{\mathrm{g}} \leq T_{\mathrm{g}}^{\max } \\
& P \leq P^{\max }, \quad \omega^{\min } \leq \omega \leq \omega^{\max } .
\end{array}
$$

\section{B. Tracking of the optimum steady-state}

Control schemes for WTG based on MPC or NMPC often rely on a tracking objective function, which acts as a surrogate for power optimization (see e.g. [11]). The tracking objective function penalizes the deviation of $\omega$ and $\beta$ from their wind-dependent steady-state optimal value $\omega^{*}(W)$ and $\beta^{*}(W)$. This section shows that the tracking approach is a quadratic approximation of (9) taken at the optimum steadystate, provided that the correct wind-dependent weighting matrix is used.

The $2^{\text {nd }}$-order Taylor expansion of the Lagrange term in (9) at the optimum wind-dependent steady-state $x^{*}(W)$ reads:

$$
\begin{aligned}
&-\frac{1}{2} \rho A C_{P} W^{3} \approx-\frac{1}{2} \rho A C_{P}^{\max } W^{3} \\
&-\frac{1}{2} \Delta X^{T} \nabla^{2}\left(\frac{1}{2} \rho A C_{P} W^{3}\right)_{\left(x=x^{*}\right)} \Delta X
\end{aligned}
$$

where $\Delta X=X-X^{*}(W)$, and $\nabla^{2}$ the Hessian operator. As a result, the cost function in (9) can be approximated as:

$$
\Phi_{\text {track }}=\int_{t}^{t+T_{\mathrm{c}}} \frac{1}{2}\left(X-X^{*}(W)\right)^{T} Q(W)\left(X-X^{*}(W)\right) d \tau,
$$

with

$$
Q(W)=-\left.\frac{1}{2} \rho A W^{3} \nabla^{2} C_{P}\right|_{X=X^{*}(W)} .
$$

Since $C_{P}$ is concave in $\omega, \beta$ in the region of interest, $Q(W)$ is positive definite and the cost function (10) can be construed as a tracking cost for the optimal wind-dependent steadystate reference $X^{*}(W)$.
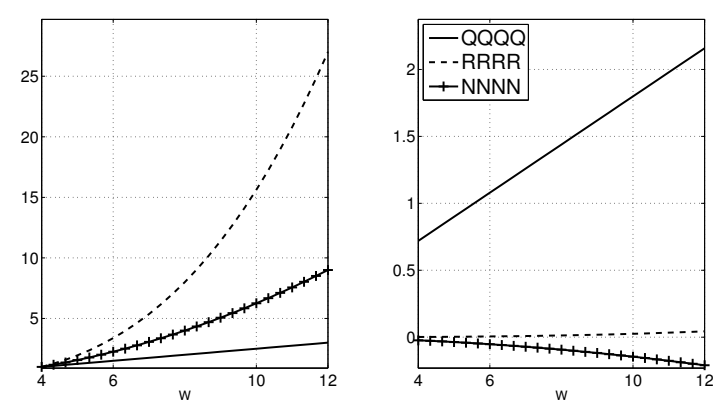

Fig. 4. Example of reference-tracking weights for problem (10), relative (left graph) and absolute (right graph)

Using equation (3), it follows that the wind-dependent weighting matrix $Q$ reads:

$$
Q(W)=-\frac{1}{2} \rho A W\left[\begin{array}{cc}
R^{2} \frac{\partial^{2} C_{P}}{\partial \omega^{2}} & W R \frac{\partial^{2} C_{P}}{\partial \omega \partial \lambda} \\
W R \frac{\partial^{2} C_{P}}{\partial \lambda \partial \omega} & W^{2} \frac{\partial^{2} C_{P}}{\partial \lambda^{2}}
\end{array}\right] .
$$

As an illustrative example, Fig. 4 displays the entries of $Q(W)$ (scaled to the MW unit) corresponding to the $C_{P}$ coefficient displayed in Figure 1. The strong, nonlinear dependence of $Q$ on the wind speed, and the large contribution of the off-diagonal terms $Q_{12}=Q_{21}$ can be observed.

The reference-tracking approach is often used in the literature with a diagonal weighting matrix $Q$ held constant within a given control region (see e.g. [11], [14]). In the light of the results presented in this section, such choices are weakly justified, albeit they are sensible from a purely practical point of view. Indeed, since the $C_{P}$ function is not strongly sensitive to deviations from the optimum rotor speed and pitch angle, a tracking of the steady-state optimum is often sufficient to yield a good performance in term of power generation.

The concern, however, pertains to the heuristic involved in tuning the reference-tracking NMPC objective function. In contrast, using a formulation in the form (9) or its local approximation (10) provides a systematic way of building the power optimization problem, and entails an objective function having a well-defined physical unit (e.g. MW), which can be easily translated into economical terms, and balanced against the competing control objectives (regulation of the power output, reduction of the pitch activity, alleviation of the structural fatigue)

\section{Gauss-Newton Hessian approximation}

The aerodynamic coefficient $C_{P}$ is typically obtained from blade-momentum theory [3]. In the context of NMPC, such models are, however, overly expensive, and are therefore interpolated. The solutions to NMPC are commonly computed numerically, based on Newton-type techniques, whose guaranteed convergence require the Lipschitz continuity of the Hessian of the underlying optimization problem.

In the context of NMPC, the interpolation of the aerodynamic coefficients must yield sufficiently smooth functions. Moreover, to achieve NMPC in real time, it is highly desirable to use a cost function that fits in the framework of 
Gauss-Newton Hessian approximation, where a cost function of the form:

$$
\Phi=\int_{t}^{t+T_{\mathrm{c}}} \frac{1}{2}\|F(X, U)\|^{2} d \tau
$$

is required, where $F \in \mathbb{R}^{2 n}$ is null at the system optimum steady-state, and $\|\cdot\|$ is the $L_{2}$-norm. In this paper, it is proposed to construct a surrogate model for $C_{P}$ in the form of the Lagrange term in (11). The following interpolation is proposed:

$$
\begin{aligned}
& C_{p}^{\max }-C_{p}(\lambda, \beta) \approx\left\|F_{\Theta}(\lambda, \beta)\right\|^{2}, \\
& F_{\Theta}(\lambda, \beta)=\Theta \zeta\left(\frac{\lambda-\lambda^{*}}{\Delta \lambda_{\max }}, \frac{\beta-\beta^{*}}{\Delta \beta_{\max }}\right)
\end{aligned}
$$

where the function:

$$
\zeta(a, b)^{T}=\left[\begin{array}{lllll}
a & b & \ldots & a^{n} & b^{n}
\end{array}\right] \in \mathbb{R}^{2 n}
$$

is a polynomial basis. The Lagrange term in (9) can then be approximated by:

$$
\begin{aligned}
-P_{\text {aero }}=\frac{1}{2} \rho A W^{3}\left(C_{p}^{\max }-C_{p}\right)-\frac{1}{2} \rho A V^{3} C_{p}^{\max } & \equiv \\
\frac{1}{2} \rho A W^{3}\left(C_{p}^{\max }-C_{p}\right) & \approx \frac{1}{2} \rho A\left\|W^{\frac{3}{2}} F_{\theta}\right\|^{2} .
\end{aligned}
$$

By construction, $\left(\lambda^{*}, \beta^{*}\right)$ achieves $\left\|F_{\Theta}\right\|^{2}=0$, hence $\left\|F_{\Theta}\right\|^{2}$ and $-P_{\text {aero }}$ share the exact same minimum. Matrix $\Theta \in$ $\mathbb{R}^{2 n \times 2 n}$ is obtained by solving the fitting problem:

$$
\min _{\Theta} \sum_{k=0}^{N}\left\|\frac{1}{2} F_{\Theta}\left(\lambda_{k}, \beta_{k}\right)^{T} F_{\Theta}\left(\lambda_{k}, \beta_{k}\right)-\left(C_{p}^{\max }-C_{P}\left(\lambda_{k}, \beta_{k}\right)\right)\right\|^{2},
$$

where $\lambda_{k}, \beta_{k}$ is a set of relevant points in the $C_{P}$ table, and the scaling factors $\Delta \lambda_{\max }, \Delta \beta_{\max }$ are chosen as $\Delta \lambda_{\max }=\max _{k}\left|\lambda_{k}-\lambda^{*}\right|, \Delta \beta_{\max }=\max _{k}\left|\beta_{k}-\beta^{*}\right|$. In the NMPC scheme,

$$
C_{P}^{\max }-\left\|F_{\Theta}(\lambda, \beta)\right\|^{2} \approx C_{p}(\lambda, \beta)
$$

is used as a surrogate for $C_{P}$ in the model dynamics (1). Observe that $n=1$ yields a $C_{P}$ interpolation similar to the second-order approximation (10). The look-up table for $C_{t}$ can be interpolated using standard polynomial fitting.

Though the interpolation of $C_{P}$ (12) can yield an excellent accuracy around the optimal pitch angle and tip-speed ration, obtaining the same level of accuracy over a large range of $\lambda, \beta$ using a single polynomial interpolation is difficult. This problem can arguably be tackled using an approach based on spline surfaces in the fitting problem (13), which provide the necessary smoothness for Newton-type optimization, while offering a very high level of interpolation accuracy.

A fitting algorithm for surface spline in the form of (13) and providing the required features is however, to our best knowledge, yet to be developed. Moreover, in the context of real-time NMPC, the on-line implementation of the interpolation algorithm must be computationally highly efficient at providing the function value and its sensitivities. The development of the necessary algorithms is the object of future work.

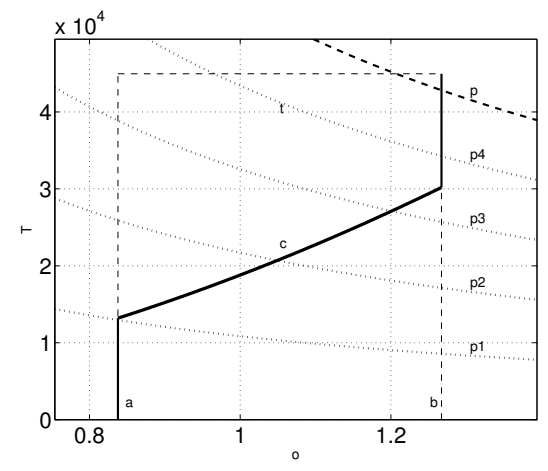

Fig. 5. Example of operational strategy for a 5 MW WTG. The generator torque and the rotor speed are subject to box constraints. The thick nonsmooth curve displays the steady-state optimal relationship between the rotor speed and generator torque.

\section{Operational CONSTRAINTS \& REgularization}

The rotor speed and generator torque of MMW-WTG are subject to soft operational constraints, i.e. constraints that ought not be excessively violated (see Fig. 5). These operational constraints are detailed next.

1) A minimum rotor speed $\omega^{\text {cut-in }}$ is imposed, below which the operation of the generator is not feasible, or economically not viable. A maximum rotor speed (or rated speed) $\omega^{\text {rated }}$ is imposed for the sake of structural fatigue, noise regulations, and power electronics limitations.

2) A maximum generator torque $T_{\mathrm{g}}^{\max }$ is imposed due to the electrical limitations of the generator and power electronics. A negative torque is clearly prohibited.

3) The electrical power is limited to its rated value, i.e. the maximum steady-state power output for which the WTG is designed.

This set of constraints is collectively referred to as the operational strategy (see e.g. [8], and Fig. 5). When using a reference-tracking NMPC scheme, the operational strategy is implemented by providing an ad-hoc wind-dependent reference for $\omega$ [11]. If using a power-maximizing NMPC scheme of the form (9), the operational strategy is best handled in the form of box constraints:

$$
\begin{aligned}
\omega^{\text {cut-in }} \leq \omega & \leq \omega^{\text {rated }} \\
P & \leq P_{\text {rated }} \\
0 \leq T_{\mathrm{g}} & \leq T_{\mathrm{g}}^{\text {max }}
\end{aligned}
$$

Because of the system perturbations and state estimation error, in the context of MPC, the recursive feasibility of some of the state constraints is not guaranteed. The rotor speed $\omega$ is subject to wind perturbations and model errors, hence constraints (14)-(15) are the most problematic. In the following, a relaxation of the constraints is proposed:

$$
g(X, U)-S \leq 0, \quad S \geq 0
$$

where $g$ lumps together constraints (14)-(15). The timedependent vector $S \in \mathbb{R}^{2}$, labelled slack variables (see e.g. [1], p. 131), is assimilated as a control variable in the NMPC 
scheme, and penalized in objective function such that any constraint violation yields an extra cost. The NMPC scheme (9) is modified to:

$$
\min _{U, X, S}-\frac{1}{2} \rho A \int_{t}^{t+T_{\mathrm{c}}} C_{P} W^{3}+\underbrace{\frac{1}{2}\left(S+S_{0}\right)^{T} Q_{\mathrm{S}}\left(S+S_{0}\right)}_{=\Pi_{S}} d \tau
$$

s.t. $\quad(1)$,

$$
\begin{aligned}
& -\dot{\beta}^{\max } \leq \dot{\beta} \leq \dot{\beta}^{\max }, \quad 0 \leq T_{\mathrm{g}} \leq T_{\mathrm{g}}^{\max } \\
& g(X, U)-S \leq 0, \quad S \geq 0 .
\end{aligned}
$$

where $Q_{\mathrm{S}} \in \mathrm{R}^{2}$ is a positive definite diagonal matrix, $S_{0} \in \mathrm{R}^{2}$, and $S_{0}^{T} Q_{\mathrm{S}}>0$. It can be verified that $\Pi_{S}$ imposes a mixed $L_{1} / L_{2}$ penalty on constraints violations. Indeed, by choosing $S_{0}^{T} Q_{\mathrm{S}}>0$, the penalty $\Pi_{S}$ is identical to:

$$
\Pi_{S}=\int_{t}^{t+T_{\mathrm{c}}}(\underbrace{\left(\frac{1}{2} S^{T} Q_{\mathrm{S}} S\right.}_{L_{2} \text { penalty }}+\underbrace{S_{0}^{T} Q_{\mathrm{S}} S}_{L_{1} \text { penalty }}+\mathrm{cst} .) d \tau,
$$

therefore formulation (18) allows for imposing the $L_{1}$ penalty $S_{0}^{T} Q_{\mathrm{S}} S$ on the constraint violations, while avoiding the introduction of a rank-deficiency in the Hessian of the NMPC problem. The $L_{1}$ penalty is crucial in order to impose a gradient in $\Pi_{S}$ at $S=0$, and balance the incentive of the powermaximizing objective function to violate the constraints in order to gain performance. In fact, parameters $Q_{S}$ and $S_{0}$ ought to be chosen such that the $L_{1}$ weight $Q_{S} S_{0}$ is always larger than the Lagrange multipliers associated to the hard constraints (14)-(15) would be. In practice, $Q_{\mathrm{S}} S_{0}$ simply ought to be chosen sufficiently large. However, since the available aerodynamic power is proportional to $W^{3}$, it is useful to make the weight $Q_{\mathrm{S}} S_{0}$ also proportional to $W^{3}$, so as to keep a constant balance between the slack penalty and the incentive of the power-optimizing objective function to violate constraints.

\section{A. Regularization of the generator torque}

The regularization of the control inputs is beneficial for the numerical stability and efficiency of the NMPC scheme, though it is necessarily performed at the expense of pure performance. A sensible choice of input regularization is therefore important. Unfortunately, the heuristic design of an appropriate regularization often requires numerous simulations.

However, an important control objective is the achievement of some level of constancy of the generated power, which is likely to be economically strongly rewarded in the future. Moreover the grid code demands that the variations of the generated power over time obey some specific dynamics (see e.g. [12] for an overview on power requirements).

Introducing the generator variation $\dot{T}_{\mathrm{g}}$ as a control input, and using the generated power variations demanded by grid code requirements $\dot{P}_{\text {elec }}^{\text {ref }}$, the following regularization of the generator torque is proposed:

$$
\left\|\dot{P}-\dot{P}^{\mathrm{ref}}\right\|^{2}=\left\|\eta r_{\mathrm{g}}^{-1}\left(\dot{T}_{\mathrm{g}} \omega+T_{\mathrm{g}} \dot{\omega}\right)-\dot{P}^{\mathrm{ref}}\right\|^{2},
$$

and arguably offers an economically meaningful metric while providing the necessary regularization of the generator torque.

\section{CONCLUSION \& FURTHER CONSIDERATION}

This paper has presented an economic NMPC scheme for WTG control which does not suffer from the classical drawbacks of economic NMPC. The proposed formulation is analyzed, and related to the more classical reference-tracking approach for WTG control. A real-time implementation of the NMPC scheme proposed here is presented in detail in the companion paper [4].

A crucial extension of the NMPC scheme presented here is the translation of the regularization of the pitch rate $\dot{\beta}$ and pitch acceleration $\ddot{\beta}$, and the WTG structural oscillations into economical terms as well. Such extension is the object of future developments.

\section{ACKNOWLEDGMENTS}

This research was supported by Research Council KUL: PFV/10/002 Optimization in Engineering Center OPTEC, GOA/10/09 MaNet; Flemish Government: IOF/KP/SCORES4CHEM, FWO: PhD/postdoc grants and projects: G.0320.08 (convex MPC), G.0377.09 (Mechatronics MPC); IWT: PhD Grants, projects: SBO LeCoPro; Belgian Federal Science Policy Office: IUAP P7 (DYSCO, Dynamical systems, control and optimization, 2012-2017); EU: FP7-EMBOCON (ICT-248940), FP7-SADCO ( MC ITN-264735), ERC ST HIGHWIND (259 166), Eurostars SMART, ACCM.

\section{REFERENCES}

[1] S. Boyd and L. Vandenberghe. Convex Optimization. University Press, Cambridge, 2004

[2] A.E. Bryson and Y.-C. Ho. Applied Optimal Control. Wiley, New York, 1975.

[3] Burton, T., Sharpe, D., Jenkins, N. and Bossanyi, E. Wind Energy Handbook. John Wiley and Sons, Ltd, Chichester, UK, 2002.

[4] S. Gros, M. Vukov, and M. Diehl. A Real-time MHE and NMPC Scheme for the Control of Multi-Mega Watts Wind Turbines. In Conference on Decision and Control (submitted), 2013.

[5] T.G. Hovgaard, L. F. S. Larsen, J. B. Jorgensen, and S. Boyd. MPC for Wind Power Gradients Utilizing Forecasts, Rotor Inertia, and Central Energy Storage. In European Control Conference, 2013.

[6] A. Koerber and R. King. Nonlinear Model Predictive Control for Wind Turbines. In Proc.EWEA, 2011.

[7] M. Mirzaei, M. Soltani, N. K. Poulsen, and H. H. Niemann. An MPC approach to individual pitch control of wind turbines using uncertain LIDAR measurements. In European Control Conference, 2013.

[8] I. Munteanu, A.I. Bract, N.-A. Cutululis, and E. Ceangă. Optimal Control of Wind Energy Systems. Springer, 2007.

[9] James B. Rawlings and Rishi Amrit. Optimizing Process Economic Performance using Model Predictive Control. In Proceedings of NMPC 08 Pavia. 2009.

[10] D. Schlipf, A. Rettenmeier, and P.W. Cheng. Model of the Correlation between Lidar Systems and Wind Turbines for Lidar Assisted Control. In 16th International Symposium for the Advancement of BoundaryLayer Remote Sensing, Boulder, CO, 2012.

[11] D. Schlipf, D.J. Schlipf, and M. Kuehn. Nonlinear Model Predictive Control of Wind Turbines Using LIDAR. Wind Energy, 2012.

[12] B. Singh and S. Singh. Wind Power Interconnection into the Power System: A Review of Grid Code Requirements. The Electricity Journal, 22:5463, 2009

[13] M. Soliman, O.P. Malik, and D.T. Westwick. Multiple-Model MIMO Predictive Control for Variable Speed Variable Pitch WindTurbines. In Proc. American Control Conference, 2010.

[14] M. D. Spencer, K. A. Stol, C. P. Unsworth, J. E. Cater, and S. E. Norris. Model predictive control of a wind turbine using short-term wind field predictions. Wind Energy, 2012.

[15] B. Srinivasan, S. Palanki, and D. Bonvin. Dynamic optimization of batch processes: I. Characterization of the nominal solution. Comp. Chem. Eng., 44:1-26, 2003. 